\title{
GNGA FOR GENERAL REGIONS: SEMILINEAR ELLIPTIC PDE AND CROSSING EIGENVALUES
}

\author{
JAY L. HINEMAN AND JOHN M. NEUBERGER
}

\begin{abstract}
We consider the semilinear elliptic PDE $\Delta u+f(\lambda, u)=0$ with the zero-Dirichlet boundary condition on a family of regions, namely stadions. Linear problems on such regions have been widely studied in the past. We seek to observe the corresponding phenomena in our nonlinear setting. Using the Gradient Newton Galerkin Algorithm (GNGA) of Neuberger and Swift, we document bifurcation, nodal structure, and symmetry of solutions. This paper provides the first published instance where the GNGA is applied to general regions. Our investigation involves both the dimension of the stadions and the value $\lambda$ as parameters. We find that the so-called crossings and avoided crossings of eigenvalues as the dimension of the stadions vary influences the symmetry and variational structure of nonlinear solutions in a natural way.
\end{abstract}

\section{INTRODUCTION}

We are interested in the connections between the linear problem

$$
\begin{aligned}
\Delta u+\lambda u & =0 \text { in } \Omega \\
u & =0 \text { on } \partial \Omega
\end{aligned}
$$

and superlinear elliptic zero-Dirichlet boundary value problems of the form

$$
\begin{aligned}
\Delta u+f(\lambda, u) & =0 \text { in } \Omega \\
u & =0 \text { on } \partial \Omega,
\end{aligned}
$$

where $\Delta$ is the Laplacian operator, $\Omega \subset \mathbb{R}^{N}$ is in general a piece-wise smooth bounded region, and $f$ satisfies certain hypotheses detailed in Section 2 . In particular, we take $f: \mathbb{R} \times \mathbb{R} \rightarrow \mathbb{R}$ to be defined by $f(\lambda, u)=\lambda u+u^{3}$, where $\lambda$ is a real parameter, and $\Omega=\Omega_{r} \subset[0,1] \times[0,1]$ to be a stadion as per [8]. Precisely, we investigate (1) and (2) on a discrete collection $\Gamma$ of such regions $\Omega_{r}$ (also referred to in the literature as stadia) defined by

$$
\begin{aligned}
\Omega_{r} & =B_{r}((r, r)) \cup((r, 1-r) \times(0,2 r)) \cup B_{r}((1-r, r)) \\
\Gamma & =\left\{\Omega_{r}: r \in\{0.10,0.11, \ldots, 0.30\}\right\} .
\end{aligned}
$$

(see Figure 1). One may consider such regions as examples, whereby our results demonstrate the applicability of the Gradient Newton Galerkin Algorithm (GNGA) to so-called general regions.

The linear problem on the family of regions $\Omega_{r}$ has been widely investigated, beginning with the seminal papers [7] and [8]. Subsequently, interesting phenomena relating to the region and/or the eigenvalues of the linear problem (1) on these regions has been documented. The most noteworthy physical phenomena is the 


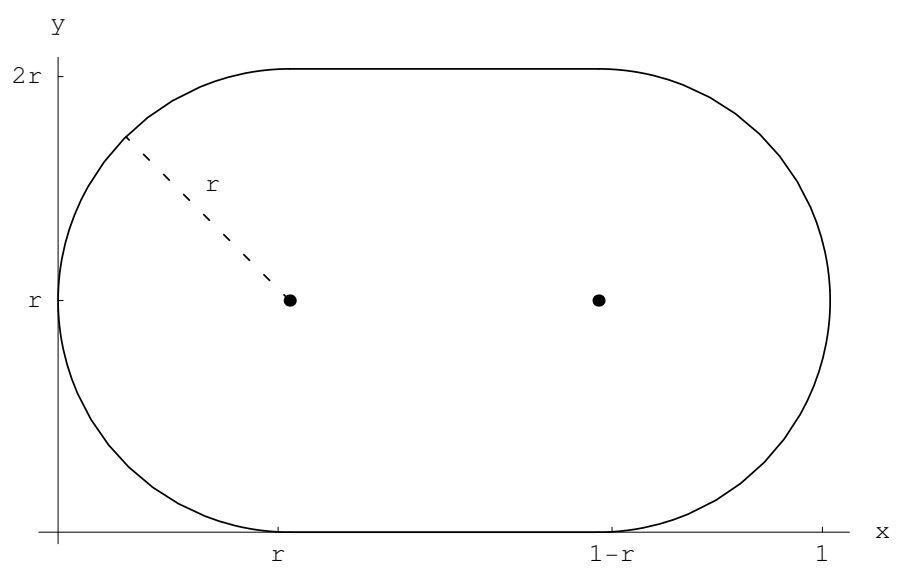

Figure 1. A region $\Omega$ in the family $\Gamma$ of stadions. The parameter $r \in\left(0, \frac{1}{2}\right]$ determines the radius of the endcap semicircles, and thus the dimensions of the connecting rectangle. For our numerical experiments, $r$ takes on finitely many equally spaced values (3).

relationship between stochastic behavior of the quantum mechanical system and the chaotic and quasi-periodic behavior of the classical system (for a concise summary of these issues see [6]). In this article we are interested in the persistence of linear properties in the nonlinear case. In particular, we observe and report the influence of the crossings and avoided crossings of eigenvalues on the bifurcation diagram for PDE (2). Correspondingly, for our nonlinear problem we demonstrate that symmetry swapping and non-swapping can occur at multiple eigenvalues and, respectively, at near-multiple eigenvalues.

In [13], the GNGA was developed to investigate existence, multiplicity, nodal structure, bifurcation, and symmetry of problems of the form (2). In that work, the region $\Omega$ was taken to be the unit square; here we perform analogous experiments on stadions. Since our implementation of the GNGA requires an orthonormal basis of eigenfunctions of the Laplacian as input, experiments on the square are easily performed using Fourier series with a basis of sine functions. In this article, we face the considerable challenge of first obtaining eigenfunctions (solutions to the linear problem (1)) numerically. In [6], this was done using essentially the inverse power method with deflation. Using ARPACK, we recreate and perhaps improve upon the results in [6] and are successful in obtaining a sufficiently large basis of such functions. This algorithmic variant of the Arnoldi process called the Implicitly Restarted Arnoldi Method (see [16]) works well on large sparse matrices such as those associated with the discretization of the Laplacian, requiring only a userprovided subroutine giving the action of the linear map.

In Section 2 we briefly state the hypothesis of the nonlinear problem, provide some background, and describe the GNGA. In Section 3 we describe the ARPACK implementation used to generate the necessary basis of eigenfunctions needed to execute GNGA. We provide some results concerning the symmetry of eigenfunctions and projections. Also, we summarize our linear numerical results for (1), which essentially duplicate those found in [6]. In Section 4 we provide results about the symmetry of solutions to the nonlinear PDE. Section 5 details our nonlinear 
experiments for (2), in which the phenomena revealed in the linear experiments are reflected. In particular, we provide portions of 8 bifurcation diagrams, corresponding to before and after a crossing and an avoided crossing. The associated contour plots demonstrate that the symmetry swapping and the nodal shape changing well observed in the linear case in fact persists in the nonlinear case. We include some numerical evidence for the analysis of symmetry done in Section 4 . Section 6 provides some short concluding remarks.

\section{VARiational Formulation and GNGA}

2.1. New Challenges. Our variational algorithm requires that we have an orthonormal collection of eigenfunctions which spans some subspace of $\mathcal{L}^{2}(\Omega)$. Recall that the eigenvalues of $-\Delta$ with zero-Dirichlet boundary condition on any piecewise smooth boundary satisfy

$$
0<\lambda_{1}<\lambda_{2} \leq \lambda_{3} \leq \cdots \rightarrow \infty .
$$

We designate the corresponding eigenfunctions by $\left\{\psi_{i}\right\}_{i \in \mathbb{N}}$, taken to be normalized in $\mathcal{L}^{2}=\mathcal{L}^{2}(\Omega)$ and of course orthogonal in both the Sobolev space $H=H_{0}^{1,2}(\Omega)$ and in $\mathcal{L}^{2}$, with inner products

$$
\langle u, v\rangle_{H}=\int_{\Omega} \nabla u \cdot \nabla v d x \text { and }\langle u, v\rangle_{2}=\int_{\Omega} u v d x,
$$

respectively. For relevant theorems, definitions, and an explanation of this notation, see [2]. The references [9] and [15] are also good resources for information on Sobolev spaces. For our stadions $\Omega=\Omega_{r}$, we can no longer use the well-known doubly indexed basis of sine functions. In the next section we describe how we obtain reasonable numerical approximations to basis elements on our decidedly non-square region where there is no known closed-form solution.

2.2. Hypotheses on $f$. We consider specific assumptions which have lead to existence theorems of sign-changing solutions (see [3], [4], [5]). As in [13], in this paper we focus on the case where $f$ is superlinear and subcritical, and in particular, defined by $f(u)=\lambda u+u^{3}$. We wish to emphasize that although infinitely many solutions have been proven to exist for various special cases, e.g., when $N=1$, $f$ is odd, or $\Omega$ is a ball in $\mathbb{R}^{N}$, in the general case only 3 nontrivial solutions are currently proven to exist (see for example [14]).

2.3. The Gradient Newton Galerkin Algorithm-GNGA. We provide a brief outline of the underlying variational machinery. We define the action (energy) functional $J: H \rightarrow \mathbb{R}$ by

$$
J(u)=\int_{\Omega}\left\{\frac{1}{2}|\nabla u|^{2}-F(u)\right\},
$$

where $F(u)=\int_{0}^{u} f(s)$ for all $u \in H$ defines the primitive of $f$. The appropriate hypotheses imply that $J$ is well-defined on all of $H$ (by the Sobolev Embedding Theorem, see [2]) and twice differentiable (see [1]). Moreover, $u$ is a solution of (2) if and only if $J^{\prime}(u)(v)=0$ for all $v \in H$. We refer the reader to [1] and [9] for the regularity theory proving this assertion. Since we are searching for critical points of $J$ we need the following identities for $J$ with $u, v, w \in H$ :

$$
J^{\prime}(u)(v)=\int_{\Omega}\{\nabla u \cdot \nabla v-f(u) v\}
$$


and

$$
J^{\prime \prime}(u)(v, w)=\int_{\Omega}\left\{\nabla v \cdot \nabla w-f^{\prime}(u) v w\right\} .
$$

For more precise details about GNGA see [13] and [12].

To summarize the GNGA, one performs the following steps:

(1) Define region $\Omega$, nonlinearity $f$, and step size $\delta$.

(2) Obtain orthonormal basis $\left\{\psi_{k}\right\}_{k=1}^{M}$ for a sufficiently large subspace $G \subset H$.

(3) Choose initial coefficients $a=a^{0}=\left\{a_{k}\right\}_{k=1}^{M}$, set $u=u^{0}=\sum a_{k} \psi_{k}$, and set $n=0$.

(4) Loop over $n$ until $\sqrt{g \cdot g} \approx\|\nabla J(u)\|$ is sufficiently small.

(a) Calculate $g=g^{n+1}=\left(J^{\prime}(u)\left(\psi_{k}\right)\right)_{k=1}^{M} \in \mathbb{R}^{M}$ (gradient vector).

(b) Calculate $A=A^{n+1}=\left(J^{\prime \prime}(u)\left(\psi_{j}, \psi_{k}\right)\right)_{j, k=1}^{M}$ (Hessian matrix).

(c) Compute $\chi=\chi^{n+1}=A^{-1} g$ by implementing least squares.

(d) Set $a=a^{n+1}=a^{n}-\delta \chi$ and update $u=u^{n+1}=\sum a_{k} \psi_{k}$.

(e) Calculate $\operatorname{sig}(A(a))$ and $\hat{J}(a)$ if desired.

The signature $\operatorname{sig}(A(a))$ of a solution is taken to be the number of negative eigenvalues of the Hessian of that solution. The signature provides us with the Morse index of a solution whenever the solution is nondegenerate (has an invertible Hessian), provided that $M$ is sufficiently large. The parameter $\delta \in(0,1]$ is the step size for damped Newton's method; generally undamped Newton's method (with $\delta=1)$ suffices.

\section{The Linear Problem, ARPACK, and Basis Generation}

3.1. The Linear Problem. The linear problem (1) is solved using ARPACK, since the standard discretization of the negative Laplacian map results in a large, sparse matrix $(L)$. To make our system visually intuitive and to provide ARPACK with knowledge of the region, we first generate a region file with $(n+1)^{2}$ values. A value of 1 represents a point of our grid that is interior to the stadion boundary, whereas a value of 0 represents a point which is exterior. To find the eigenvalues of the matrix $L$, ARPACK requires a user-provided subroutine for repeatedly calculating the action of multiplying $L$ times a vector $v$ whenever requested. The region file read in by our ARPACK driver is processed in the following way.

In this explanation, we take $i \in\left\{1, \ldots, n_{r}\right\}$, where $n_{r}$ is the number of interior grid points, and $j \in\left\{1, \ldots,(n+1)^{2}\right\}$, where there are a total of $(n+1)^{2}$ grid points in our $n \times n$ discretization of the unit square. We define the maps $s:\left\{1, \ldots, n_{r}\right\} \rightarrow$ $\left\{1, \ldots,(n+1)^{2}\right\}$ and $t:\left\{1, \ldots,(n+1)^{2}\right\} \rightarrow\left\{0,1, \ldots, n_{r}\right\}$ by

$$
s_{i}=j \text { where the } i^{t h} \text { interior grid point is the } j^{\text {th }} \text { overall grid point, }
$$

and

$$
\left\{\begin{aligned}
t_{j} & =0 \text { if the } j^{t h} \text { grid point is exterior, otherwise } \\
t_{j} & =i \text { where } s_{i}=j .
\end{aligned}\right.
$$

Thus $t$ and $s$ are inverses for interior grid points, i.e.,

$$
t_{s_{i}}=i, i=1, \ldots, n_{r} \text {, and } s_{t_{j}}=j \text { if the } j^{\text {th }} \text { grid point is interior. }
$$

Via the second difference formula, we see that the action of the discretized negative Laplacian $L$ on a given $v \in \mathbb{R}^{n_{r}}$ can be computed componentwise by

$$
(L v)_{i}=n^{2}\left[4 v_{i}-\left(v_{t s_{i}-1}+v_{t s_{i}+1}+v_{t s_{i}-(n+1)}+v_{t s_{i}+(n+1)}\right)\right],
$$


where the additonal value $v_{0}=0$ is used to enforce boundary conditions.

We note that our methodology here allows us to use the GNGA on any twodimensional bounded region. We could go so far as to create our region file based upon a figure drawn with black pixels corresponding to 1s and white pixels corresponding to 0s. This methodology would in theory allow for solving many linear and semilinear problems on different (possibly related) regions in quick succession.

The family $\Gamma$ (3) we have chosen to study was selected so that the corresponding interiors (represented by 1's in the region file) are symmetric about the horizontal and vertical medians (our primary choice was $n=100$ ). If these regions are not symmetric it will introduce errors into our numerics and our theoretical results will not hold. The choice of $n$ is arbitrary; experiments have been performed with larger choices of $n$. Since our discretization is symmetric, the output of our ARPACK driver is a basis of eigenfunctions of four symmetries, namely, $(+1,+1),(+1,-1)$, $(-1,+1)$, and $(-1,-1)$, corresponding to oddness and eveness about the medians $x=\frac{1}{2}$ and $y=r$ (see Section 3.2).

3.2. Symmetry of Eigenfunctions. A Mathematica notebook was written to sort the first few eigenfunctions on stadions in $\Gamma$ by their symmetry. This way we can clearly see the two linear phenomena that we are interested in, those being crossings and avoided crossings. Loosely, crossings (double eigenvalues) can occur when the symmetry of the corresponding eigenfunctions is different - conversely, avoided crossings (nearly double eigenvalues) can only occur when the symmetry type is the same. If we naïvely plot the first 18 (say) eigenvalues for the family $\Gamma$ and connect the $k^{\text {th }}$ ones we produce an inaccurate picture as far as symmetry goes. For a more precise definition of these phenomena and and description of the implementation, see Section 3.3.

The sorting of eigenfunctions and eigenvalues relies on projections; the deeper underlying structure involves representation theory (see [11]).

Definition 3.2.1. Define operators $P_{\mu, \nu}: V \rightarrow V$ by

$$
P_{\mu, \nu}[u(x, y)]=\frac{u(x, y)+\mu u(1-x, y)+\nu u(x, 1-y)+\mu \nu u(1-x, 1-y)}{4},
$$

where $u: \Omega \rightarrow \mathbb{R}$ and $\mu, \nu \in\{-1,+1\}$. It is apparent that $P_{\mu, \nu}$ with $\mu, \nu \in\{-1,+1\}$ is a linear operator. Here we take $V$ to be either the vector space $\mathcal{L}^{2}$ or $H$, but with a suitable adjustment corresponding to discrete indexing instead of function evaluation, the space could also be taken to be $\mathbb{R}^{n_{r}}$. If $P_{\mu, \nu} u=u$, we say that $u$ has $(\mu, \nu)$ symmetry.

We are examining the symmetry of functions in two dimensions; we do this through examining the function as a function of one variable and do what is usual there, i.e., consider eveness and oddness. We denote being even in one dimension by a ' +1 ' and being odd by a ' -1 '.

The symmetry of $\mathcal{L}^{2}$ functions plays an important role in the expected symmetry of a bifurcation. The set of symmetries of eigenfunctions forms a subset of the set of all symmetries of solutions to the nonlinear equation (2). In particular, the primary branches of nontrivial solutions bifurcating from the trivial branch contain solutions with symmetries in this restricted subset (see Section 5). Some relevant and useful facts about the symmetry of eigenfunctions of (1) and general properties of the operators $P_{\mu, \nu}$ are summarized in the following theorem statement: 
Theorem 3.2.1. (1) Given $u \in \mathcal{L}^{2}$, the function $P_{\mu, \nu}[u]$ has $(\mu, \nu)$ symmetry. (2) Given any function $u \in \mathcal{L}^{2}$ we have

$$
\sum_{\mu, \nu \in\{-1,+1\}} P_{\mu, \nu}[u(x, y)]=u(x, y) .
$$

(3) $P_{\mu, \nu}$ is a projection.

(4) For all $\left(\psi_{i}, \lambda_{i}\right)$ which satisfy $-\Delta \psi_{i}=\lambda_{i} \psi_{i}$ on the stadion region with zeroDirichlet boundary condition, so does $P_{\mu, \nu}[\psi]$ with $\mu, \nu \in\{-1,+1\}$.

(5) The function $u \in \mathcal{L}^{2}$ has symmetry type $(\mu, \nu)$ if and only if for all $m, n \in$ $\{-1,+1\}$ the relation $P_{m, n}[u(x, y)]=\delta_{m \mu} \delta_{n \nu} u(x, y)$ holds.

The groups that represent both rigid motions of the region and rigid motions of solutions to (2) and (1) are nice enough in our case to keep our discussion of symmetry simple. In Chapter 4, we will discuss the underlying group that gives rise to the projection operators developed above.

3.3. Numerical Computations of Crossings and Avoided crossings. We need precise definitions of the two linear phenomena that we're searching for before we can proceed.

Definition 3.3.1. The $n^{\text {th }}$ eigenvalue branch of $(\mu, \nu)$ symmetry is the set of ordered pairs $\left(r, \lambda_{n}\right)$ where $r \in(0,1 / 2]$ and $\lambda_{n}$ is the $n^{\text {th }}$ eigenvalue satisfying (1) corresponding to an eigenfunction of $(\mu, \nu)$ symmetry.

Definition 3.3.2. We define a crossing as an ordered pair $(r, \lambda)$ where an eigenvalue branch of symmetry $(\mu, \nu)$ and another eigenvalue branch of symmetry $(m, n)$ (where $(\mu, \nu) \neq(m, n))$ intersect.

That is to say, there exist eigenfunctions of distinct symmetry that correspond to the same eigenvalue for some radius $r$.

Definition 3.3.3. An avoided crossing is a value $r^{*}$ where the $n^{\text {th }}$ and $(n+1)^{\text {st }}$ eigenvalue branches of common symmetry $(\mu, \nu)$ contain ordered pairs $\left(r, \lambda_{n}\right)$ and $\left(r, \lambda_{n+1}\right)$ such that $\lambda_{n+1}-\lambda_{n}$ achieves a local minimum at $r=r^{*}$.

For each of the first few eigenfunctions for a given stadion we compute the projections $P_{+1,+1}, P_{+1,-1}, P_{-1,+1}$ and $P_{-1,-1}$ at four points $\left(x_{0}, y_{0}\right),\left(1-x_{0}, y_{0}\right),\left(x_{0}, 2 r-\right.$ $\left.y_{0}\right)$ and $\left(1-x_{0}, 2 r-y_{0}\right)$, where the origin is identified with the point $(1 / 2, r)$. By Theorem 3.2.1 (5), we can say that an eigenfunction is of a specific $(\mu, \nu)$ symmetry if the projection $P_{\mu, \nu}$ performed at the four points yields the value at the point $\left(x_{0}, y_{0}\right)$. After this is done, we connect the $k^{t h}$ eigenvalue for each stadion in $\Gamma$ with a line style (thick, thin, dash, dot) according to its symmetry. The result of this procedure is displayed in Figure 2.

We observe that the property that we are interested in is that to the left or right of a crossing we have two branches with different symmetries which change at a crossing. Conversely, in the case of an avoided crossing we have two branches of the same symmetry which fail to cross and consequently do not change symmetry. The crossing and avoided crossing in Figure 2 serve as a starting point for our nonlinear experiments. 


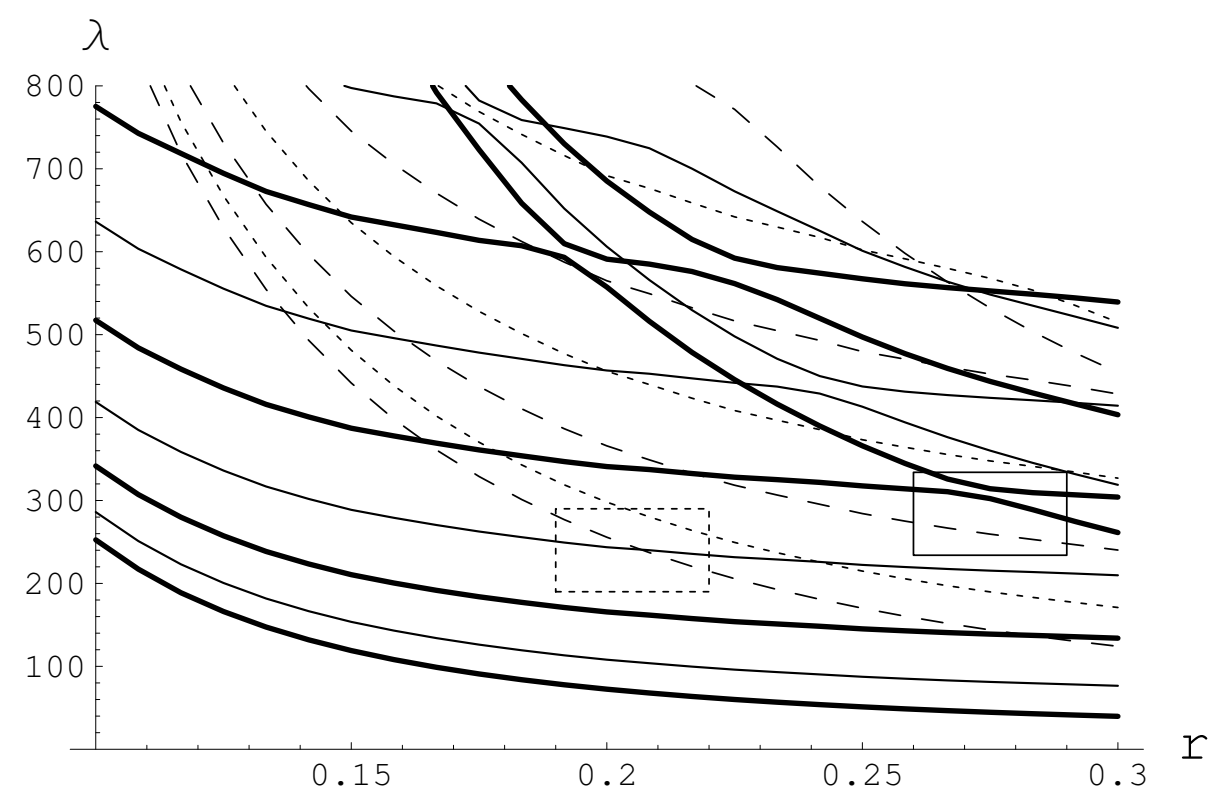

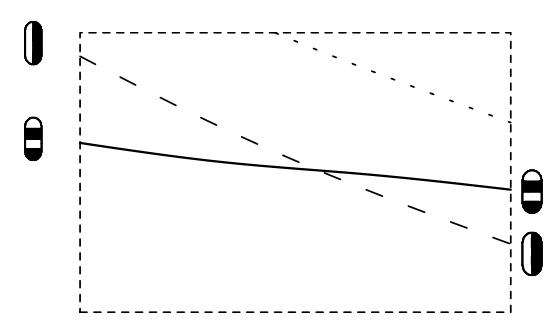

(a) Enlargement of the particular crossing that we numerically investigate.

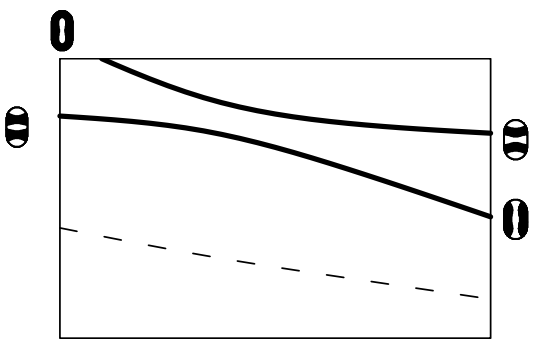

(b) Enlargement of the particular avoided crossing that we investigate.

Figure 2. A plot of the first 18 eigenvalues for (1). The eigenvalues $\lambda_{k}$ are plotted as a function of the radius $r$, with the four line styles corresponding to the symmetry of the associated eigenfunctions. In the crossing graphic Figure 2(a), the small contour plots demonstrate that with our choice of ordering for the eigenvalues, the symmetry types of corresponding eigenfunctions remain constant along each branch. In the avoided crossing graphic Figure 2 (b), the contour plots demonstrate that a change in the nature of the eigenfunctions ocurrs on the featured branches, despite the fact that the symmetry remains constant at type $(+1,+1)$.

\section{Symmetry of Functions on $\Omega_{r}$}

We proceed in developing the expected symmetries for primary, secondary, and teritary bifurcations. We begin by considering the rigid motion of an arbitrary stadion in the plane back to itself. These motions form a group known as $\mathbb{V}_{4}$ : the 
Klein 4-group. We choose to describe this group as the set

$$
\{1, \tau, \sigma, \rho\},
$$

with the operation of composition of motions. We let 1 serve as the identity, or the rigid motion of leaving the figure fixed, whereas $\tau$ represents a reflection across the horizontal median of a stadion, $\sigma$ represents a reflection across the vertical median of a stadion, and $\rho=\tau \sigma$ is a $180^{\circ}$ rotation about the center of a stadion. The rigid motions of this group are the same as that of a rectangle.

We are interested in identifying the symmetric $\mathcal{L}^{2}$ functions from $\Omega_{r}$ into $\mathbb{R}$ which satifisy the boundary conditions of the odd PDE (1) and (2). We can represent rigid motions of these functions with the same group $\mathbb{V}_{4}$ combined with the group $\{-1,1\}$ (under multiplication) in the sense of take a direct product. That is, we define the group $G$ as the set

$$
\{1,-1\} \times\{1, \tau, \sigma, \rho\},
$$

for which we have multiplication of ordered pairs defined by $(a, b)(c, d)=(a c, b d)$. One may notice that $\mathbb{V}_{4}$ is isomorphic to $\mathbb{Z}_{2} \oplus \mathbb{Z}_{2}$ and $\{-1,1\}$ is isomorphic to $\mathbb{Z}_{2}$; both are additive groups. So, $G$ is isomorphic to $\mathbb{Z}_{2} \oplus \mathbb{Z}_{2} \oplus \mathbb{Z}_{2}$, which is clearly Abelian by construction.

All of our eigenfunctions are of one of four possible symmetries. We list explicitly those four fixed-point subspaces denoted as follows:

$$
\begin{aligned}
& s_{1}=\left\{u: P_{+1,+1}[u]=u\right\} \equiv \operatorname{im}\left(P_{+1,+1}\right) \\
& s_{2}=\left\{u: P_{-1,+1}[u]=u\right\} \equiv \operatorname{im}\left(P_{-1,+1}\right) \\
& s_{3}=\left\{u: P_{+1,-1}[u]=u\right\} \equiv \operatorname{im}\left(P_{+1,-1}\right) \\
& s_{4}=\left\{u: P_{-1,-1}[u]=u\right\} \equiv \operatorname{im}\left(P_{-1,-1}\right),
\end{aligned}
$$

where the notation 'im' denotes 'image'. One may note that $s_{1} \oplus s_{2} \oplus s_{3} \oplus s_{4}=\mathcal{L}^{2}(\Omega)$.

When a bifurcation occurs we lose symmetry - this can be described in terms of moving down the lattice of isotropy (stabilizer) subgroups of the group $G$ or as taking another projection (see Figure 4 and Table 3 ). Our special case greatly simplifies the discussion since in general we must consider conjugacy classes of isotropy subgroups. Since $\mathbb{V}_{4}$ is Abelian, conjugacy classes contain only one element. We provide in Figure 4 a lattice that includes both subgroups and fixed point subspaces. We emphasize that our results for the symmetry of solutions to (2) are mainly observational; for examples, see Section 5 .

In each of the cells in the lattice in Figure 4 we provide the generators of each isotropy subgroup and the corresponding fixed point subspace $s_{k}$ which are subspaces of $\mathcal{L}^{2}\left(\Omega_{r}\right)$. The group itself is of order eight, the first row of isotropy subgroups all have order four, the third row of isotropy subgroups all have order two, and the final row containing the identity subgroup is of order one. In a cascade of bifurcations we have primary branches bifurcating from an eigenvalue (on the trivial branch), secondary branches bifrucating from primary branches, and tertiary branches bifurcating from secondary branches. The fixed point subspaces $s_{k}$ can be organized in terms of which type of bifurcation branches (trivial, primary, secondary, or tertiary) they contain. The analogous organization can be done in terms of the isotropy subgroups. We order the fixed point subspaces in Figure 3.

In Section 5 we observe both primary and secondary bifurcations which have solutions that fall in the appropriate fixed-point spaces. We have yet to find a tertiary bifurcation or follow a tertiary branch. Based upon experiments performed in [13], 
(1) Trivial Fixed Point Space

$$
s_{0}=\{0\} .
$$

(2) Primary Fixed Point Spaces

$$
\begin{aligned}
& s_{1}=\left\{u: P_{+1,+1}[u]=u\right\}=\operatorname{im}\left(P_{+1,+1}[u]\right) \\
& s_{2}=\left\{u: P_{-1,+1}[u]=u\right\}=\operatorname{im}\left(P_{-1,+1}[u]\right) \\
& s_{3}=\left\{u: P_{+1,-1}[u]=u\right\}=\operatorname{im}\left(P_{+1,-1}[u]\right) \\
& s_{4}=\left\{u: P_{-1,-1}[u]=u\right\}=\operatorname{im}\left(P_{-1,-1}[u]\right) .
\end{aligned}
$$

(3) Secondary Fixed Point Spaces

$$
\begin{aligned}
s_{5} & =\left\{u: P_{+1,+1}[u]+P_{+1,-1}[u]=u\right\}=\operatorname{im}\left(P_{+1,+1}[u]+P_{+1,-1}[u]\right) \\
& =\operatorname{im}\left(P_{+1,+1}[u]\right) \oplus \operatorname{im}\left(P_{+1,-1}[u]\right) \\
s_{6} & =\left\{u: P_{+1,+1}[u]+P_{-1,+1}[u]=u\right\}=\operatorname{im}\left(P_{+1,+1}[u]+P_{-1,+1}[u]\right) \\
& =\operatorname{im}\left(P_{+1,+1}[u]\right) \oplus \operatorname{im}\left(P_{-1,+1}[u]\right) \\
s_{7} & =\left\{u: P_{+1,+1}[u]+P_{-1,-1}[u]=u\right\}=\operatorname{im}\left(P_{+1,+1}[u]+P_{-1,-1}[u]\right) \\
& =\operatorname{im}\left(P_{+1,+1}[u]\right) \oplus \operatorname{im}\left(P_{-1,-1}[u]\right) \\
s_{8} & =\left\{u: P_{-1,+1}[u]+P_{+1,-1}[u]=u\right\}=\operatorname{im}\left(P_{-1,+1}[u]+P_{+1,-1}[u]\right) \\
& =\operatorname{im}\left(P_{-1,+1}[u]\right) \oplus \operatorname{im}\left(P_{+1,-1}[u]\right) \\
s_{9} & =\left\{u: P_{+1,-1}[u]+P_{-1,-1}[u]=u\right\}=\operatorname{im}\left(P_{+1,-1}[u]+P_{-1,-1}[u]\right) \\
& =\operatorname{im}\left(P_{+1,-1}[u]\right) \oplus \operatorname{im}\left(P_{-1,-1}[u]\right) \\
s_{10} & =\left\{u: P_{-1,+1}[u]+P_{-1,-1}[u]=u\right\}=\operatorname{im}\left(P_{-1,+1}[u]+P_{-1,-1}[u]\right) \\
& =\operatorname{im}\left(P_{-1,+1}[u]\right) \oplus \operatorname{im}\left(P_{-1,-1}[u]\right) .
\end{aligned}
$$

(4) Tertiary Fixed Point Spaces

$$
\begin{aligned}
s_{11} & =\left\{u: P_{+1,+1}[u]+P_{+1,-1}[u]+P_{-1,+1}[u]+P_{-1,-1}[u]=u\right\} \\
& =\operatorname{im}\left(P_{+1,+1}[u]+P_{+1,-1}[u]+P_{-1,+1}[u]+P_{-1,-1}[u]\right) \\
& =\operatorname{im}\left(P_{+1,+1}[u]\right) \oplus \operatorname{im}\left(P_{+1,-1}[u]\right) \oplus \operatorname{im}\left(P_{-1,+1}[u]\right) \oplus \operatorname{im}\left(P_{-1,-1}[u]\right) \\
& =\mathcal{L}^{2}(\Omega)
\end{aligned}
$$

FIGURE 3. Fixed point spaces for: 1. the trivial branch; 2. primary branches; 3 . secondary branches; 4 . tertiary branches.

[12], however, we expect that tertiary solutions will also fall into the appropiate fixed point subspace indicated in Table 3 . One can justify the fixed point subpaces in Figure 3 by using basic properties of the projection operators $P_{\mu, \nu}$. For example, if $\mu, \nu, m, n \in\{-1,1\}$ then $P_{\mu, \nu}$ and $P_{m, n}$ commute. Furthermore, if $\mu \neq m$ and $\nu \neq n$, then the operator $P_{\mu, \nu}+P_{m, n}$ is itself a projection.

\section{The Nonlinear Problem: A Crossing and an Avoided Crossing}

For a given radius $r$, and hence a given region $\Omega=\Omega_{r}$, we are interested in how solutions to (2) vary as the parameter $\lambda$ varies; this is the subject of bifurcation theory. Briefly, due to the continuous dependence of the problem on $\lambda$, one expects to find continuous branches of solutions. We define our branches to be of a single symmetry type, as listed in Figure 4. On occasion, branches intersect one 


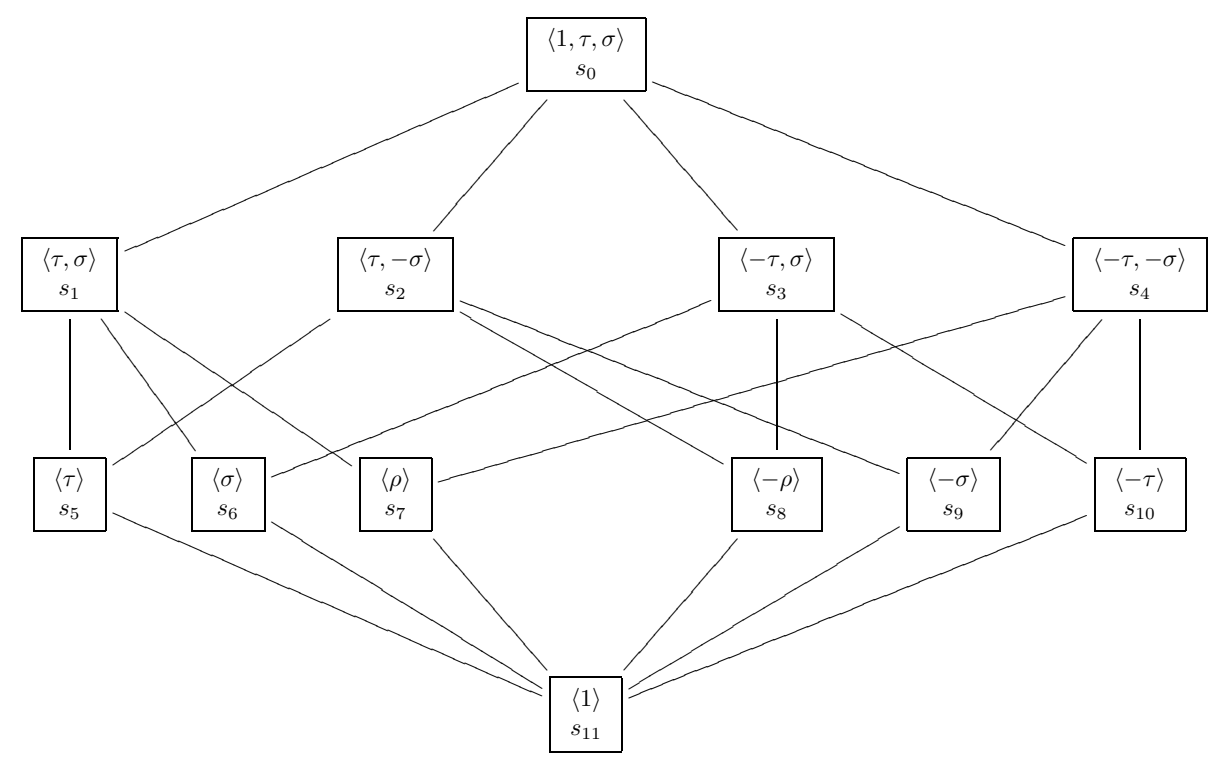

Figure 4 . The lattice of isotropy subgroups of the group $G$ and fixed point spaces defined in Figure 3. We have simplified our ordered pair notation so that our lattice of 12 symmetry types is visible on a single page (we use generator notation for the same reason). Here we write, for example, $(-1, v)$ as $-v$ and $(1, v)$ as $v$.

another; such points of interesection are called bifurcation points. There are many reasonable ways to represent portions of bifurcation diagrams. We have chosen to first fix a "generic" point in the domain. This is a point not lying on any line of symmetry, where also none of the solutions of particular interest have a zero amplitude. Plotting the value of solutions evaluated at this generic point versus the bifurcation parameter $\lambda$ results in graphics where branches are clearly separated. There other reasonable choices of $y$-coordinate for these plots, such as the value of the functional $J$ evaluated at the solution, or a suitable norm of the solution. Further information is given by choosing the line style of the plotted branches to correspond to the symmetry type of the solutions lying on those branches.

For brevity, we restrict the report of our investigations to regions $\Omega_{r}$ with $r \in$ $\{.19, .20, .21, .22\}$, where we have the crossing of the $4^{\text {th }}$ and $5^{\text {th }}$ eigenvalues with $(-1,+1)$ and $(+1,-1)$ symmetry, and $r \in\{.26, .27, .28, .29\}$, where we have an avoided crossing of the $8^{\text {th }}$ and $9^{\text {th }}$ eigenvalues with $(+1,+1)$ symmetry. Thus, we will present a total of 8 bifurcation diagrams.

Figure 5 contains 4 bifurcation diagrams corresponding to the crossing eigenvalue in Figure 2(a), for $r \in\{.19, .20, .21, .22\}$. The first two diagrams occur before the crossing, while the second two occur afterwards. In each of the diagrams, the $y$-axis is the value of solutions to (2) evaluated at the fixed generic point in the domain $\Omega_{r}$, while the $x$-axis is the bifurcation parameter $\lambda$. We use the same line styles for the primary branches as were used in Figure 2. Here, we display primary branches bifurcating from the trivial solution (which has amplitude zero) at $\lambda=\lambda_{4}$ and $\lambda=\lambda_{5}$, respectively, for each of the four consecutive radii in our discrete 
collection. Contour plots corresponding to the solutions of (2) lying on these two primary branches, for each of the four different regions and two different values of the parameter $\lambda$ can be found in Figure 6. In that figure and elsewhere, we use the notation ' $\widetilde{\psi}_{k}$ ' to denote a solution to the nonlinear equation (2) which lies on a primary branch bifurcating at $\lambda_{k}$. Such solutions 'resemble' the corresponding eigenfunction $\psi_{k}$; they are of the same symmetry type and have similar nodal structure. The level curves in these contourplots were chosen for clarity; positive levels correspond to white curves on top of a black background, while negative levels are depicted by black curves on top of a white background. Figure 6 shows that the primary bifurcation branches do in fact swap symmetry. Figure 8 contains plots of solutions along secondary branches, whereby one sees that there is a loss of symmetry that occurs when bifurcating to a secondary bifurcation branch. The main effect that we are interested in is that higher and lower amplitude branches actually swap both in terms of symmetry and in terms of Morse index. A comparison of Figures 8 and 9 shows that secondary branches also swap symmetry. In Figure 7, we provide a 3-dimensional plot of a solution to (2). This solution lies on the $\widetilde{\psi}_{4}$ primary branch of $(+1,-1)$ symmetry type, for $r=.19$ and $\lambda=100$.

The avoided crossing is somewhat less interesting, however the fact that these branches do not swap symmetry in the way that those in the crossing case do is noteworthy. There seems to be some sort of underlying mechanism changing the solutions in the avoided crossing (see Figure 11 and the 4 bifurcation diagrams in Figure 10). We see that the solutions change nature, that is, the arrangement of their positive and negative components changes, although the symmetry remains constant. We have not yet investigated the secondary bifurcations within the avoided crossing.

In applying the GNGA we are required to carefully provide initial guesses for Newton's method, particularly since the solutions we seek lie on branches that can be very close to each other. ARPACK's ability to generate many eigenfunctions efficiently is key; GNGA would be nearly impossible to implement on our family of regions without such a package. Another element which plays a critical role in providing initial guesses which converge to solutions on secondary branches is having a good understanding of the symmetry of a given branch. The symmetry of solutions can be understood by inspecting the nonzero terms of the solutions' eigenfunction expansion.

\section{Conclusions}

The tools from ARPACK and our GNGA code are a potent combination for solving semilinear elliptic PDE on general bounded two-dimensional regions. The exposition of the symmetry properties of the region was crucial in choosing an appropriate initial guess, since the bifurcation branches of crossing and avoided crossing are by their very nature close together. The article [11] and the forthcoming article [12] use more advanced techniques similar to ours to study the linear and the nonlinear problem on the Koch Snowflake region, a region with a fractal boundary.

We indicated in Chapter 4 that we observed the following symmetry phenomena: a) each primary bifurcation branch contains solutions in one of the $s_{1}-s_{4}$ fixed point spaces; and b) each secondary bifurcation branch contains solutions in one of the $s_{5}-s_{10}$ fixed point spaces. 
Each step of our iterative solver could include a projection, ensuring that iterates remain in the proper fixed point subspace. Generally, this is not required, as the invariance of the Newton search direction is fairly stable. From a computational point of view, restricting the set of nonzero eigenfunction expansion coefficients not only stabilizes this invariance, but reduces the number of floating point operations required to compute the search direction. Regardless, once solutions are found we can verify their symmetry automatically by numerically applying the projection before saving the output for plotting; (see Figures 6, 8, and 9).

From what we can ascertain, crossing and avoided crossings have not been previously so studied in nonlinear equations. A problem to consider would be to see how far our numerical techniques can effectively go, e.g., could we investigate nonlinear solutions corresponding to very high energy eigenvalues (8000 and higher). Physicists are interested in observing effects such as "scarring"; scars are narrow line-like regions visible on the contour plots of eigenfunctions with enhanced intensity that seem to be coming from classical periodic orbits (see [10] for more information).

The authors' wish to thank Nathan Borggren, B.S. Mathematics and Physics, Northern Arizona University, 2002, for his contributions. In particular, Borggren was the first to suggest investigating the effects of the crossing and avoided crossing of eigenvalues on related nonlinear problems using GNGA. 


\section{REFERENCES}

[1] Minimax Methods in Critical Point Theory with Applications to Differential Equations, volume 65 of Regional Conference Series in Mathematics, Providence, RI, AMS, 1986.

[2] R. Adams. Sobolev Spaces. Academic Press, 1975.

[3] Alfonso Castro, Jorge Cossio and John M. Neuberger. Sign-changing solutions for a superlinear dirichlet problem. Rocky Mountain Journal of Mathematics, (65), no. 4, 1997.

[4] Alfonso Castro, Jorge Cossio, and John M. Neuberger. A minimax principle, index of the critical point, and existence of sign-changing solutions to elliptic boundary value problems. Electronic Journal of Differential Equations, (2), 1998.

[5] Alfonso Castro, Pavel Drabek and John M. Neuberger. A sign-changing solution for a superlinear dirichlet problem, II. In Fifth Mississippi State Conference on Differential Equations and Computational Simulations, number 10, pages 101-107. Electronic Journal of Differential Equations, 2003.

[6] B. Neuberger, J. W. Neuberger and D.W. Noid. Eigenfunctions on a stadium assoicated with avoided crossings of energy levels. arXiv:math.NA/0105217v1, 2001.

[7] L. A. Bunimovich. On the ergoidc properties of some billiards. Functional Analysis Applications, (8), 1974.

[8] L. A. Bunimovich. On the ergodic properties of nowhere dispersing billiards. Communications in Mathematical Physics, (65), 1979.

[9] D. Gilbarg and N. Trudinger. Elliptic Partial Differential Equations of Second Order. Springer-Verlag, Berlin, New York, 1983.

[10] Martin C. Gutzwiller. Chaos in Classical and Quantum Mechanics, chapter Scars, or What Is Left of Classical Periodic Orbits, pages 249-253. Spring-Verlag, 1990.

[11] John M. Neuberger, N. Sieben and James W. Swift. Computing eigenfunction on the Koch's Snowflake: A new grid and symmetry. to appear: J. Comp. and App. Math., 2005.

[12] John M. Neuberger, N. Sieben and James W. Swift. GNGA for Semilinear Elliptic PDE on a Fractal Region: Symmetry and Automated Branch Following. Preprint, 2005.

[13] John M. Neuberger and James W. Swift. Newton's method and Morse index for semilinear elliptic PDEs. International Journal of Bifurcation and Chaos, (11) no. 3, 2001.

[14] John M. Neuberger. GNGA: Recent Progess and Open Problems for Semilinear Elliptic PDE. J.M. Neuberger, editor, Proc. of: Variational Methods: "Open Problems, Recent Progress, and Numerical Investigations, 2002." AMS J. Contemp. Math.. 357, AMS, Prov. R. I., 2004.

[15] J. W. Neuberger. Steepest descent for general systems of linear differential equations in Hilbert Spaces, volume 1032. Spring-Verlag, Berlin, 1983.

[16] R. B. Lehoucq, D. C. Sorensen, and C. Yang. ARPACK users' guide: Solution of large-scale eigenvalue problems with implicitly restarted Arnoldi methods. Software, Evironments, and Tools. SIAM, Philadelphia, PA, 1998.

E-mail address: jhineman@uab.edu

E-mail address: John.Neuberger@nau.edu

Department of Mathematics and Statistics, Northern Arizona University PO Box 5717, Flagstaff, AZ 86011-5717, USA 


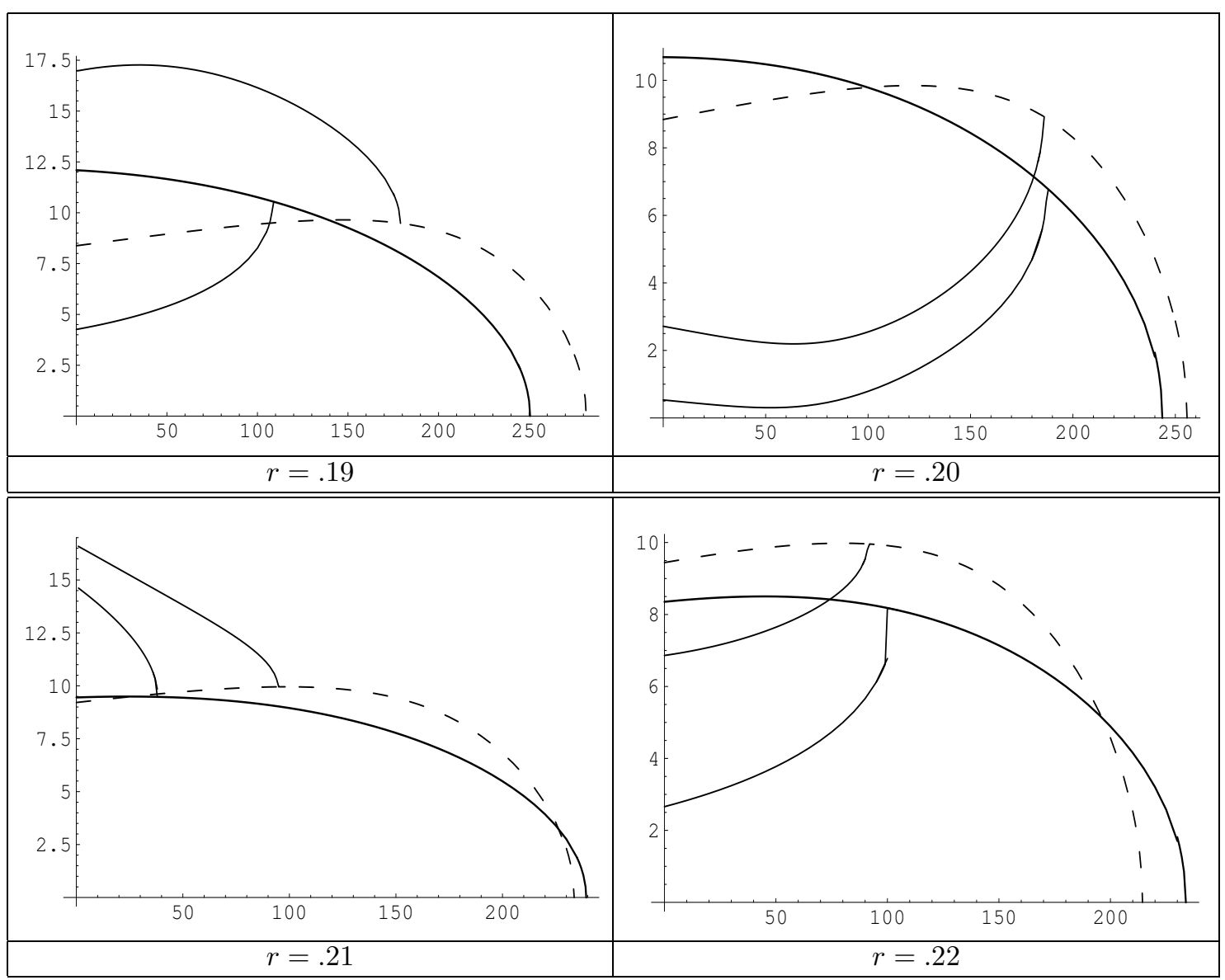

FiguRE 5. Four bifurcation diagrams depicting the primary branches bifurcating from the trivial solution (which has amplitude zero) at $\lambda=\lambda_{4}$ and $\lambda=\lambda_{5}$, for $r=.19, .20, .21, .22$. The $y$-axis is the value of solutions to (2) evaluated at the particular fixed point in the domain $\Omega_{r}$, while the $x$-axis is the bifurcation parameter $\lambda$. Figure 6 contains contour plots corresponding to the solutions of (2) lying on these two primary branches, whereby one can see the swapping of symmetry at the avoided crossing. For convenience, we term such solutions " $\widetilde{\psi}_{k}$ ", where here $k \in\{4,5\}$, since these solutions to the nonlinear equation (2) resemble the eigenfunctions $\psi_{4}$ and $\psi_{5}$ of the linear equation (1). Figures 8 and 9 contain plots of solutions along secondary branches, whereby one observes the loss of symmetry explained in part by Figure 4. A comparison shows that secondary branches also swap symmetry. 


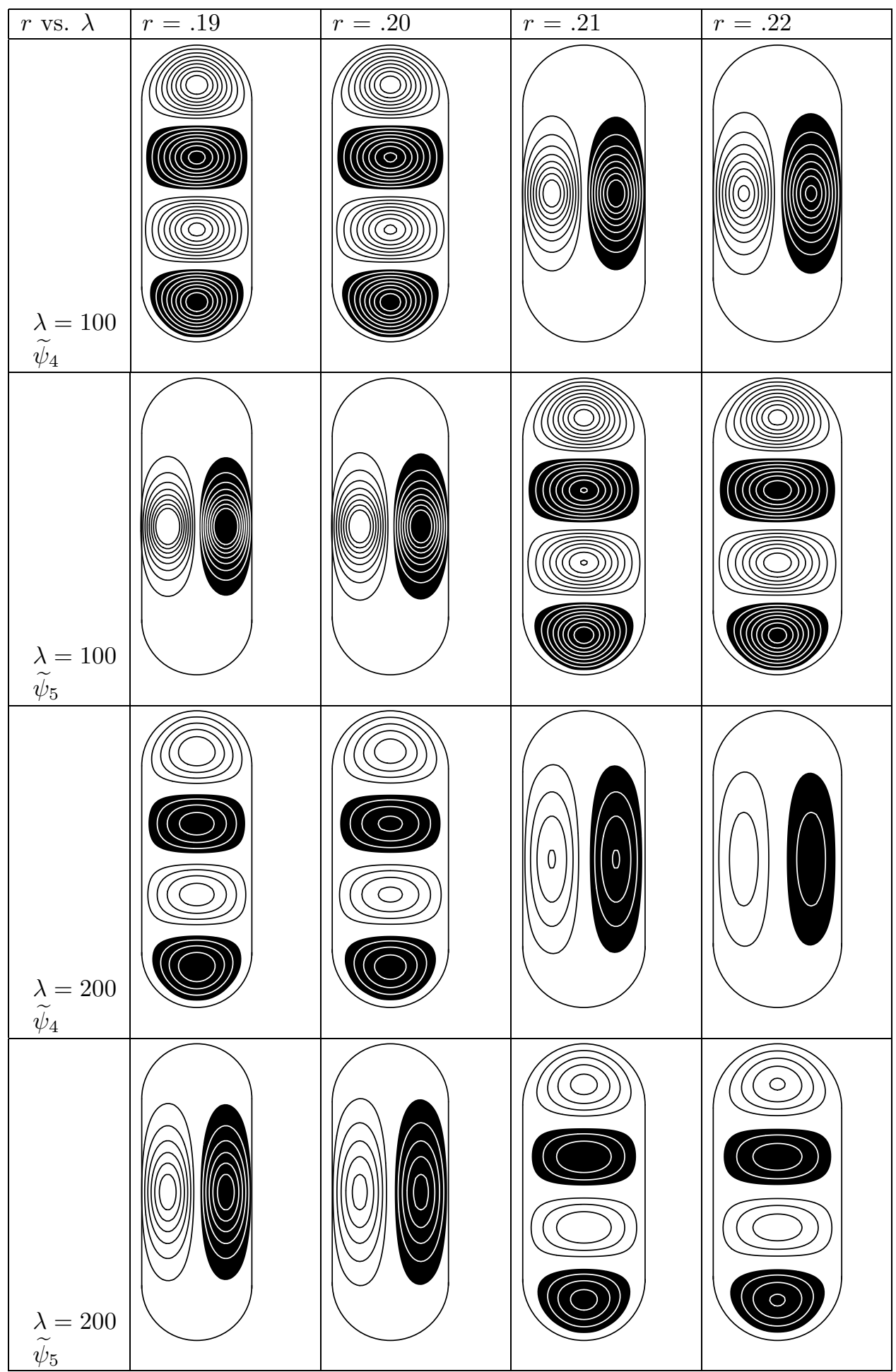

Figure 6. Solutions to the nonlinear PDE (2) near a crossing. We use the convention that $\widetilde{\psi}_{k}$ is a nonlinear solution lying on a primary branch which resembles $\psi_{k}$, where the eigenfunctions are ordered as in Figure 2. 


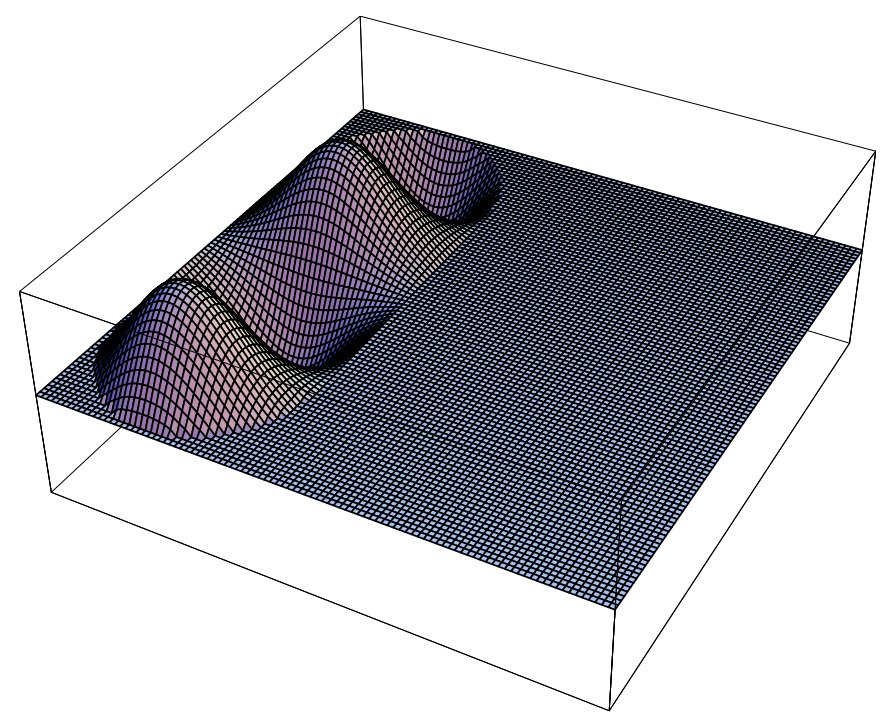

FiguRE 7. A 3-dimensional surface plot of the solution to (2) found in the upper left hand corner of Figure 6. Here, the nonlinear solution resembles closely a multiple of $\psi_{4}$, since that's the dominant mode in its eigenfunction expansion. The radius of the endcap defining the region $\Omega=\Omega_{r}$ is $r=.19$. The branch that this solution belongs to is of symmetry type $(+1,-1)$, and bifurcates from the trivial solution branch at $\lambda=\lambda_{4}$. Arbitrarily, we have selected the point on this branch at $\lambda=100$. Here, we plot the function as zero-extended to fit the larger super-domain of the unit square, as our ARPACK takes as input. 


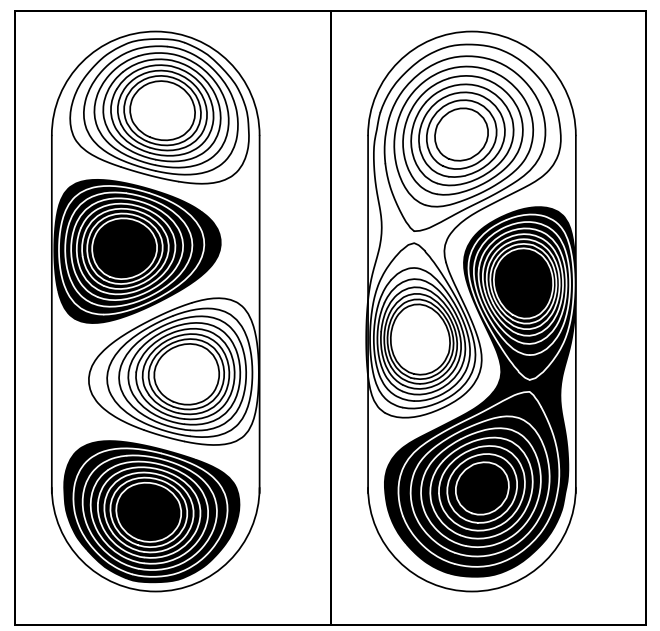

Figure 8. Contour plots of solutions to (2) of Morse index 4 and 5 , lying on secondary branches, for $r=.19$ before the crossing featured in Figure 2(a), for a fixed value of the bifurcation parameter $\lambda$. We note that these solutions have less symmetry then those in Figure 6 . We have the invariance under the projection $P_{-1,+1}+P_{+1,-1}$, indicating that the solutions are in the fixed point subspace $s_{8}$.

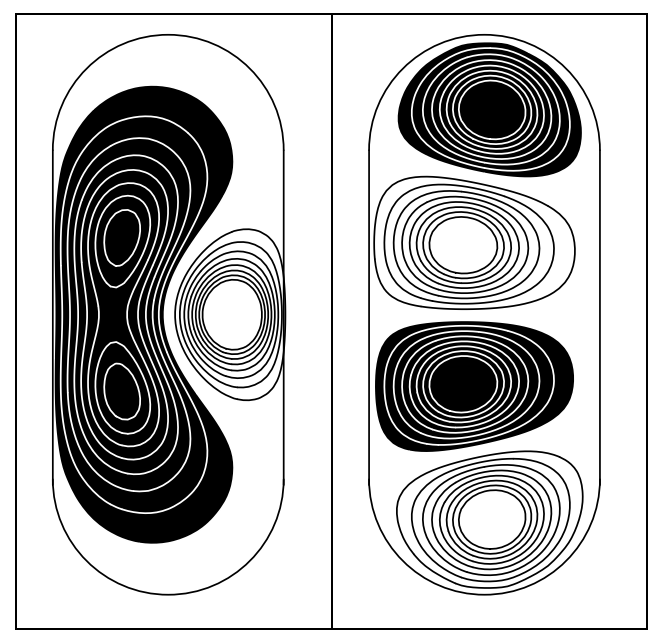

Figure 9. These contour plots are of solutions of Morse index 4 and 5 lying on secondary branches at the radius $r=.21$, after the crossing featured in Figure 2(a), for the same fixed value of the bifurcation parameter $\lambda$. Again, we notice that the solutions have less symmetry then those in Figure 6 . This time, we have invariance under the two distinct projections $P_{+1,+1}+P_{-1,+1}$ and $P_{-1,-1}+P_{+1,-1}$, hence the branches contain solutions in $s_{5}$ and $s_{10}$, respectively. 


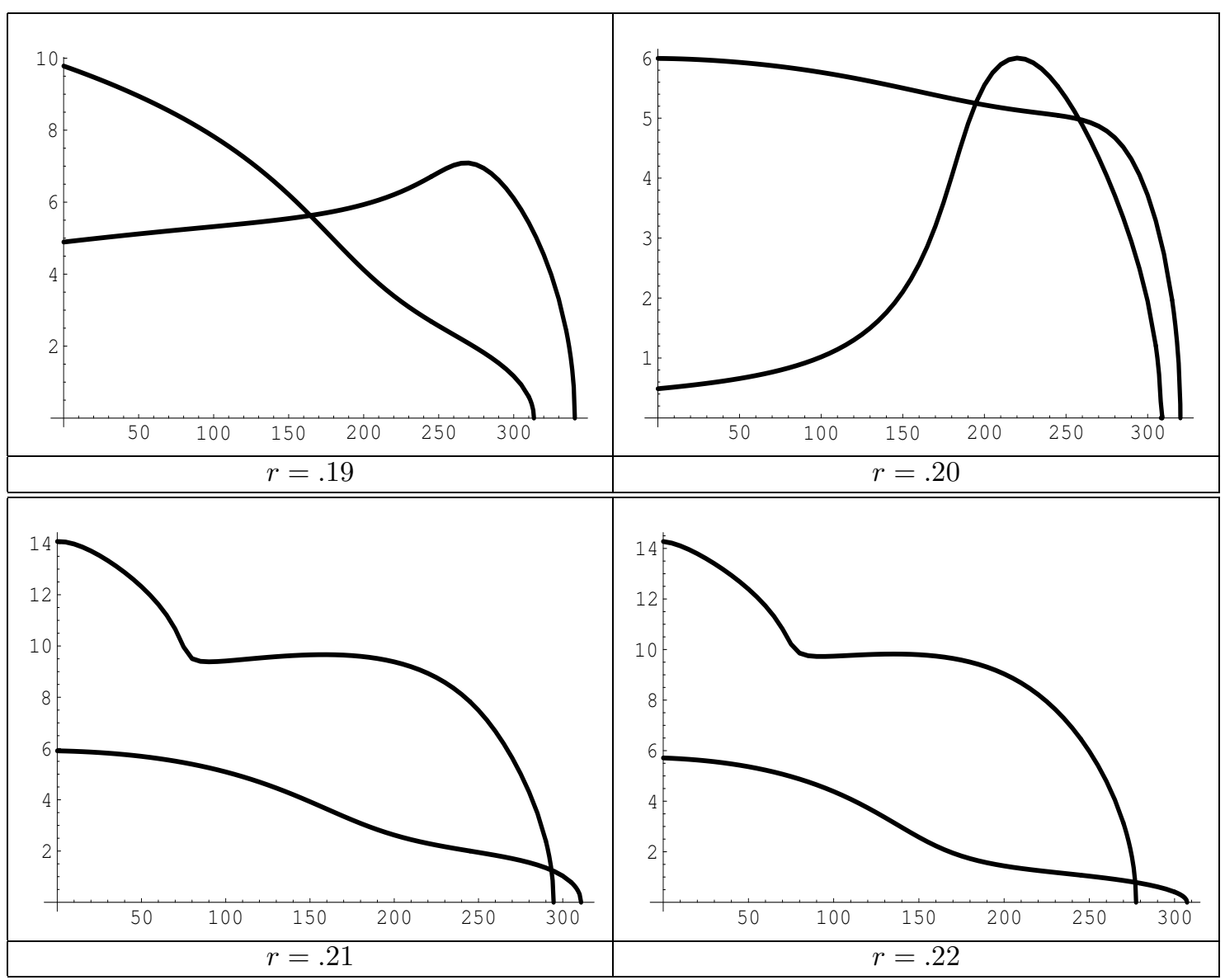

Figure 10. The four diagrams above demonstrate the effects of an avoided crossing on the primary branches bifurcating from the trivial solution at $\lambda=\lambda_{8}$ and $\lambda=\lambda_{9}$, for four consecutive radii of stadions, namely, $r=.26, .27, .28, .29$ corresponding to Figure 2(b). The contour plots displayed in Figure 11 demonstrate that although the symmetry of the solutions is constant along each branch, the nature of the solution does change at the near point of the avoided crossing. 


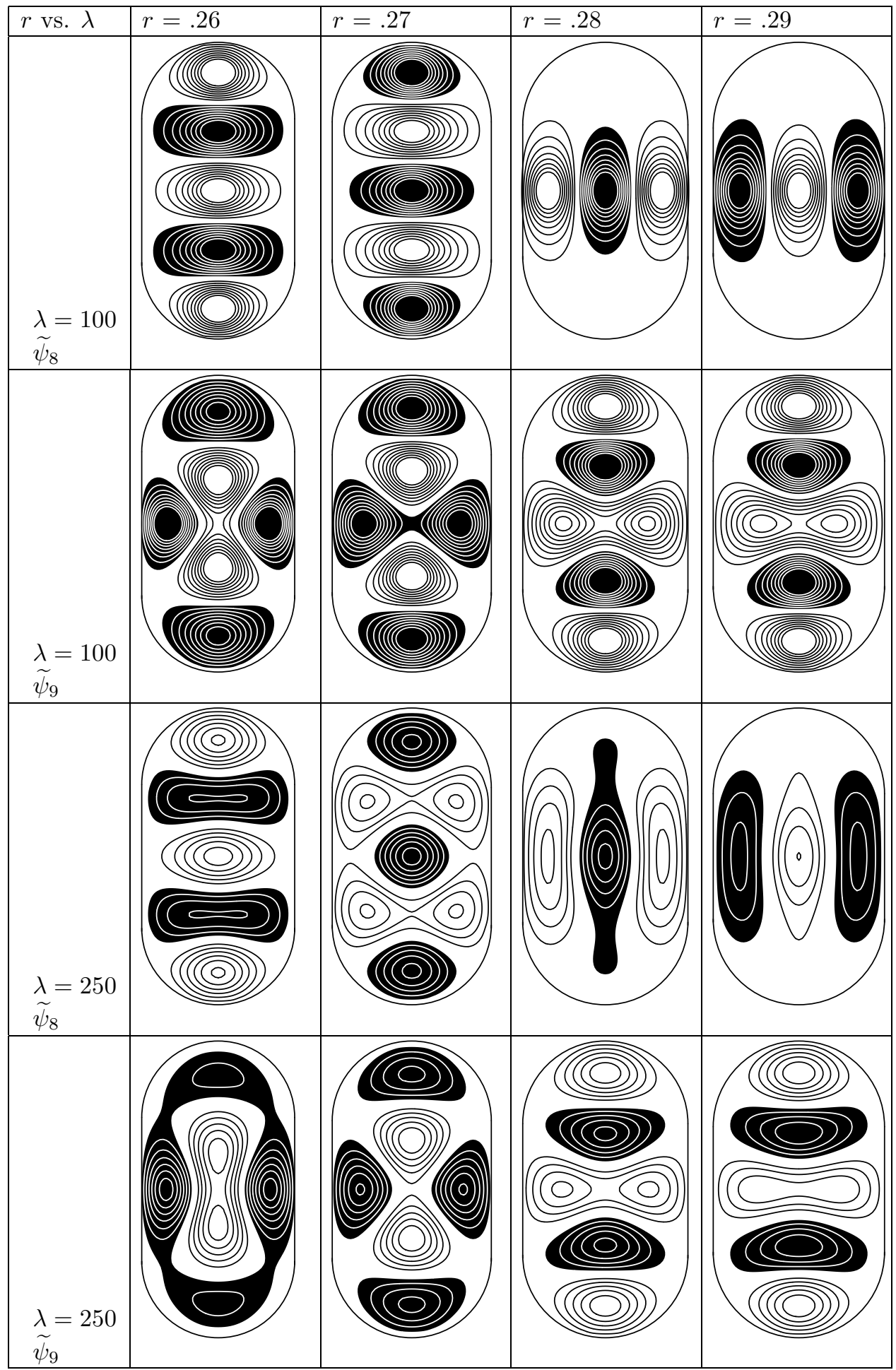

FIgURE 11. Solutions to our nonlinear PDE (2) for the avoided crossing. We see that between $r=.26$ and $r=.29$ the branches corresponding to $\lambda_{8}$ and $\lambda_{9}$ have changed in terms of nodal structure although the symmetry type is constant. As $\lambda$ increases, the symmetry type also remains constant, although the shapes of the nodal regions do change. 